\title{
Supporting Information Electronic Properties of Carbyne Chains: Experiment and Theory
}

Tristan de Boer, ${ }^{1}$ Dmitry Zatsepin, ${ }^{2,3}$ Dmitry Raikov, ${ }^{2}$ Ernst Kurmaev,${ }^{2,3}$ Anatoly F. Zatsepin ${ }^{2}$ and Alexander Moewes ${ }^{1}$

${ }^{1}$ Department of Physics and Engineering Physics, University of Saskatchewan, Saskatoon, S7N 5E2, Canada

${ }^{2}$ Institute of Physics and Technology, Ural Federal University, Ekaterinburg, 620002, Russia

${ }^{3}$ Institute of Metal Physics, Ural Branch of Russian Academy of Sciences, Ekaterinburg, 620990, Russia

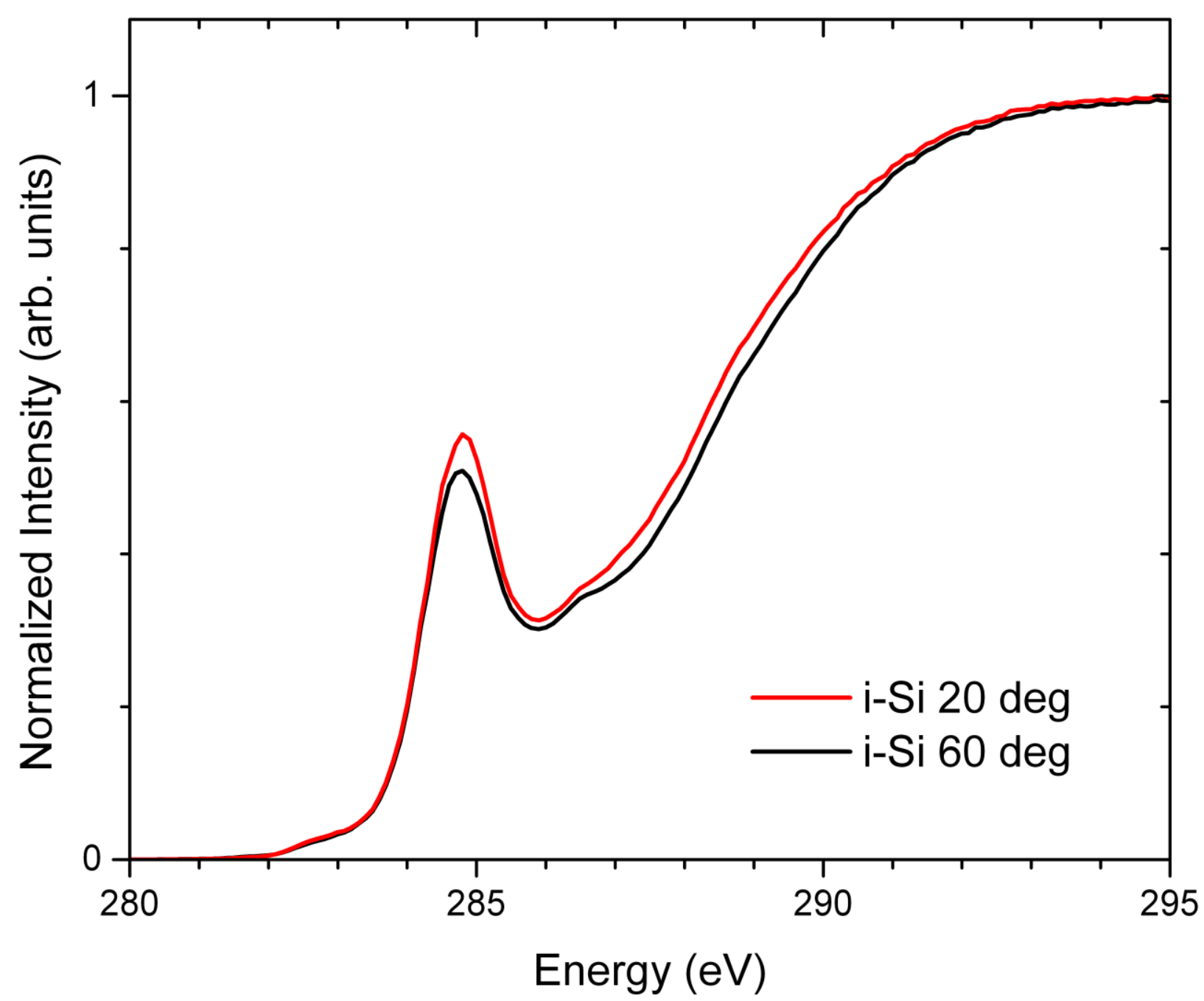

Figure S1: C K XAS TEY spectra of $200 \mathrm{~nm}$ thick LCCs grown on intrinsic Si. Spectra were taken with linear horizontal polarization of light, with the orientation of the sample surface to the incident light as shown. The spectra show negligible orientation dependence. 


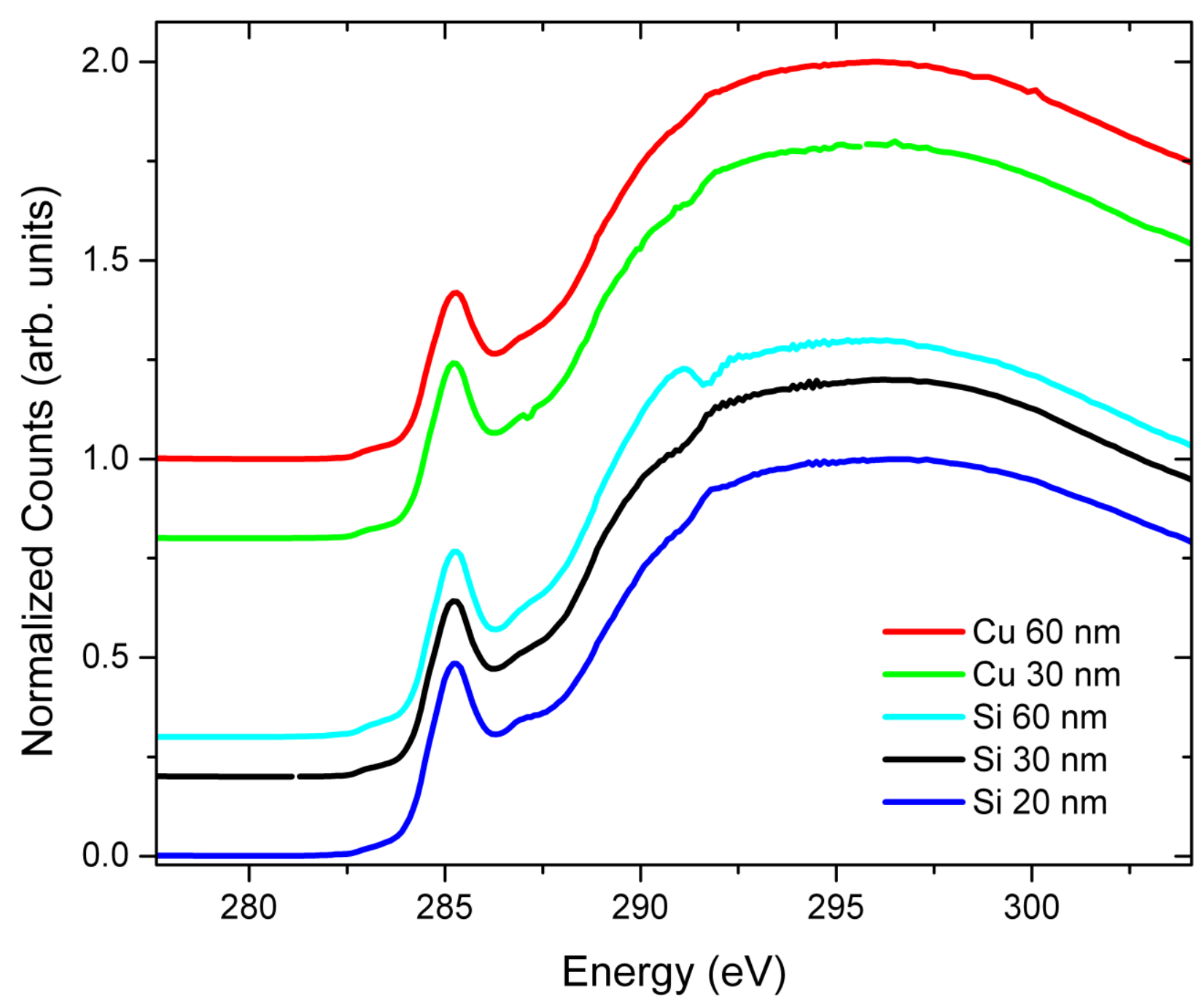

Figure S2: C K XAS TEY spectra are shown for LCCS on Si and Cu substrates, as indicated, for film thicknesses varying between 20 $\mathrm{nm}$ and $60 \mathrm{~nm}$, as indicated.

X-ray absorption spectroscopy (XAS) measurements were performed on the C K edge at the REIXS beamline at the Canadian Light Source based on carbyne linear carbon chain (LCC) samples grown on Si and $\mathrm{Cu}$ substrates. These measurements are performed by promoting core electrons to the conduction band and monitoring their subsequent decay. Linearly polarized light at an angle 60 degrees to the surface normal was used. The samples were mounted on carbon tape and XAS measurements were performed in total electron yield (TEY) mode. 